\title{
Is GRK2 a new target for cardiovascular disease?
}

\author{
Jun-ichi Oyama ${ }^{1} \cdot$ Koichi Node ${ }^{2}$
}

Received: 25 March 2018 / Accepted: 10 April 2018 / Published online: 23 May 2018

(C) The Japanese Society of Hypertension 2018

Recently, many reports have indicated that $G$ protein, G protein-coupled receptors (GPCR), and related enzymes play an important role in cardiovascular disease. GPCR are also known as seven-(pass)-transmembrane domain receptors that are coupled with G protein. GPCR kinases (GRK) are a family of protein kinases that regulate the activity of GPCR by phosphorylating their intracellular domains after their associated $G$ proteins have been released and activated. Basically, the sustained stimulation of $\beta$-adrenergic receptors ( $\beta$-AR) that transmit information via $\mathrm{G}$ protein leads to $\beta$-AR desensitization and a reduced response to $\beta$-adrenergic agonists. The desensitization is composed of two phases: an early desensitization occurring within a few seconds to several minutes, and a long-term desensitization occurring after more than $1 \mathrm{~h}$. In early desensitization, there is uncoupling of the receptor and $\mathrm{G}$ protein and disappearance of the receptor from the cell surface (sequestration). In long-term desensitization, the receptor protein is downregulated itself. In early desensitization, the receptor protein is phosphorylated by GRK or cAMP-dependent protein kinase A (PKA). Although PKA activation without receptor stimulation can phosphorylate the receptor, GRK phosphorylates only receptors activated by agonists. Homologous desensitization by GRK is considered to be an important mechanism to prevent an excessive cellular response to agonists. GRK has seven subtypes, and GRK2, which is also known as $\beta$-AR kinase ( $\beta$ ARK) 1 , is a member of the GRK family of serine/threonine protein kinases that phosphorylate and desensitize GPCR (such as adrenergic and muscarinic receptors). Upregulation or downregulation of GRK2 may

Jun-ichi Oyama

junoyama@cc.saga-u.ac.jp

1 Department of Circulation Regulation in Medicine, Saga University, Saga, Japan

2 Department of Cardiovascular Medicine, Saga University, 5-1-1 Nabeshima, Saga 849-8501, Japan occur in several pathophysiological disorders, and these changes can exacerbate cardiovascular and metabolic diseases $[1,2]$. The signaling of GRK2 in the cardiomyocytes is shown in Fig. 1.

GRK2 is an endogenous protein inhibitor of the insulin signaling pathway for glucose transport stimulation, and GRK2 inhibitors can improve glucose homeostasis and the insulin response and lead to enhanced insulin sensitivity $[2,3]$. It has also been reported that GRK2 is increased and signal transduction is suppressed in diabetes, because GRK2 antagonizes $\beta$-arrestin 2 , which is a downstream target protein [4]. Increased levels of GRK2 can decrease insulin signaling in several cell types and tissues [2]. These findings suggest that GRK2 plays an important role in the pathogenesis and deterioration of diabetes and metabolic syndrome. In addition, insulin stimulates endothelial nitric oxide synthase (eNOS) activity by Akt activation, leading to eNOS phosphorylation, NO production, and vasorelaxation. Furthermore, impaired insulin-mediated Akt/eNOSdependent signaling was recognized in diabetic murine models $[4,5]$. Therefore, GRK2 promotes diabetic endothelial dysfunction via suppression of the insulin-stimulated Akt/eNOS/NO signaling pathway, and inhibition of GRK2 expression can improve endothelial dysfunction by restoring glucose homeostasis [4-6]. The inhibition of the Akt/eNOS pathway by GRK2 in vascular endothelial cells may cause attenuated vasorelaxation. Therefore, GRK2 in endothelial cells may play an important role in abnormalities of the Akt/eNOS signal during the development of diabetic vasculopathy.

Recently, many studies have reported a relationship between GRK2 and hypertension. A link between G $\beta 3$ C825T polymorphism and hypertension was reported in population-based and case-control studies in different ethnicities in humans. Because G $\beta 3$ C825T polymorphism impairs the function of G $\beta 3$ to target GRK2 ubiquitination, the loss of G $\beta 3$ function minimizes GRK2 ubiquitination and stabilizes GRK2 [7]. In basic studies, genetic knockdown of GRK2 using a small hairpin RNA resulted in spontaneous hypertension and altered vascular GPCR 


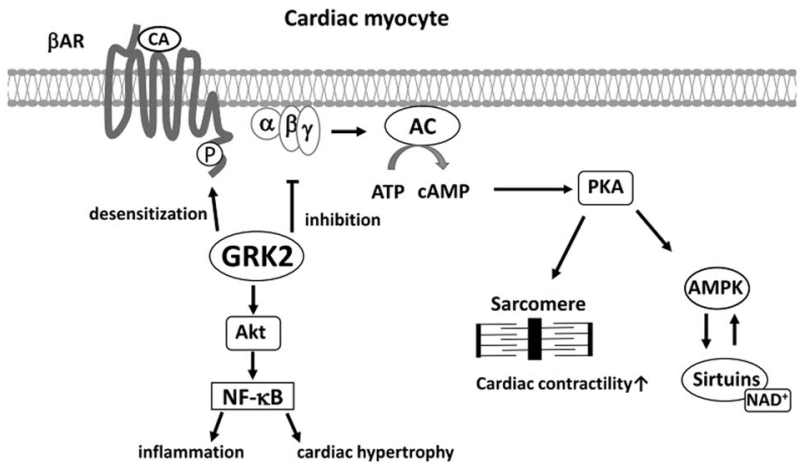

Fig. 1 Schematic drawing of GRK signaling. $\beta A R \quad \beta$-adrenergic receptor, CA catecholamines, $\mathrm{AC}$ adenylate cyclase, PKA protein kinase A, AMPK 5' adenosine monophosphate-activated protein kinase

signaling in mice [8]. In hypertensive rats, GRK2 regulates cardiac hypertrophy via upregulation of NF- $\mathrm{KB}$ activity in a phosphorylation-dependent manner. By inhibition of GRK2 activity, cardiac hypertrophy was prevented with reduced NF- $\mathrm{BB}$ transcription activity [9]. These results indicate the possibility of GRK2 as a potential therapeutic target for preventing hypertension and cardiac hypertrophy.

The study by Taguchi et al. in Hypertension Research Vol. 41, No. 4 demonstrated that GRK2 was involved in the rebound elevation of blood pressure after withdrawal of clonidine in streptozotocin-induced diabetic mice, and suppression of GRK2 activity improved diabetic endothelial dysfunction via the Akt/eNOS/NO signaling pathway [10]. Based on these findings and previous publications, GRK2 is involved in vascular disorders and hypertension in diabetes via the insulin signal, and GRK2 inhibition should improve these vicious cycles. Therefore, therapeutic strategies that target GRK2 can be effective for the treatment of hypertension in diabetes and metabolic syndrome, and this hypothesis is supported by the findings in the study by Taguchi et al.

GRK2 inhibitors have also been suggested for the treatment of heart failure (HF). HF is characterized by myocardial dysfunction and hemodynamic overload, leading to excessive neurohormonal signaling as a compensatory response. Usually, plasma catecholamine (CA) levels are first increased to maintain normal cardiac function. However, chronically elevated sympathetic nerve activity and increased CA levels lead to increased GRK2 activity in HF, resulting in subsequent desensitization and downregulation of $\beta$-ARs. Elevated GRK2 in the failing heart can attenuate the contractile response of the heart to positive inotropic agents, because upregulated GRK abrogates the contractile signaling of $\beta$-ARs. Indeed, cardiac-specific GRK2 inhibition using transgenic mice or peptide has been shown to ameliorate chronic HF symptoms and increase survival rate by improving cardiac function
$[11,12]$. These findings suggest the possibility of further improving the standard treatment of HF patients with $\beta$-blockers by adding new drugs that can inhibit GRK2.

As there has been an increase in the prevelance of ischemic heart disease and/or HF due to metabolic syndrome, it is necessary to develop a treatment strategy that targets multiple conditions, including hypertension, diabetes, and dyslipidemia. Vascular failure is involved in various cardiovascular and metabolic diseases, including diabetes, HF, and hypertension. The underlying mechanism is malfunction of $\mathrm{G}$ protein and its related factors. Especially, GRK2 has been shown to play an essential role in these diseases. It is important to further clarify the role of GRK2 and develop new drugs that can inhibit GRK2 for the treatment of $\mathrm{HF}$ in the future.

\section{Compliance with ethical standards}

Conflict of interest JO belongs to the endowed Department of Fukuda Denshi Co, Ltd. KN received remuneration including lecture fees from AstraZeneca, Astellas Pharma Inc., Nippon Boehringer Ingelheim Co, Ltd., Pfizer Inc., MSD KK, Daiichi Sankyo Company, Ltd., Kowa Pharmaceutical Co, Ltd., Takeda Pharmaceutical Co, Ltd., Novartis Pharmaceuticals Japan, Mitsubishi Tanabe Pharma Corporation, and Dainippon Sumitomo Pharma Co, Ltd. KN also received scholarship funds or donations granted by Astellas Pharma Inc., Nippon Boehringer Ingelheim Co, Ltd., Daiichi Sankyo Company, Ltd., Takeda Pharmaceutical Co, Ltd., Mitsubishi Tanabe Pharma Corporation, and MSD KK.

\section{References}

1. Freedman NJ, Lefkowitz RJ. Desensitization of G protein-coupled receptors. Recent Prog Horm Res. 1996;51:319-51.

2. Garcia-Guerra L, Nieto-Vazquez I, Vila-Bedmar R, Jurado-Pueyo M, Zalba G, Díez J, et al. G protein-coupled receptor kinase 2 plays a relevant role in insulin resistance and obesity. Diabetes. 2010;59:2407-17.

3. Usui I, Imamura T, Satoh H, Huang J, Babendure JL, Hupfeld CJ, et al. GRK2 is an endogenous protein inhibitor of the insulin signaling pathway for glucose transport stimulation. EMBO J. 2004;23:2821-9.

4. Taguchi K, Matsumoto T, Kamata K, Kobayashi T. G proteincoupled receptor kinase 2, with $\beta$-arrestin 2, impairs insulininduced Akt/eNOS signaling in ob/ob mouse aorta. Diabetes. 2012;61:1978-85.

5. Kobayashi T, Taguchi K, Yasuhiro T, Matsumoto T, Kamata K. Impairment of PI3-K/Akt pathway underlies attenuated endothelial function in aorta of type 2 diabetic mouse model. Hypertension. 2004;44:956-62.

6. Taguchi K, Hida M, Hasegawa M, Narimatsu H, Matsumoto T, Kobayashi T. Suppression of GRK2 expression reduces endothelial dysfunction by restoring glucose homeostasis. Sci Rep. 2017;7:8436.

7. Zha Z, Han XR, Smith MD, Lei QY, Guan KL, Xiong Y. Hypertension-associated C825T polymorphism impairs the function of G $\beta 3$ to target GRK2 ubiquitination. Cell Discov. 2016;2:16005. 
8. Tutunea-Fatan E, Caetano FA, Gros R, Ferguson SS. GRK2 targeted knock-down results in spontaneous hypertension, and altered vascular GPCR signaling. J Biol Chem. 2015;290:5141-55.

9. Sorriento D, Santulli G, Franco A, Cipolletta E, Napolitano L, Gambardella J, et al. Integrating GRK2 and NFkappaB in the pathophysiology of cardiac hypertrophy. J Cardiovasc Transl Res. 2015;8:493-502.

10. Taguchi K, Bessho N, Hasegawa M, Narimatsu H, Matsumoto T, Kobayashi T. Co-treatment with clonidine and a GRK2 inhibitor prevented rebound hypertension and endothelial dysfunction after withdrawal in diabetes. Hypertens Res. 2018;41:263-74.

11. Reinkober J, Tscheschner H, Pleger ST, Most P, Katus HA, Koch WJ, et al. Targeting GRK2 by gene therapy for heart failure: benefits above $\beta$-blockade. Gene Ther. 2012;19: 686-93.

12. Cannavo A, Komici K, Bencivenga L, D'amico ML, Gambino G, Liccardo D, et al. GRK2 as a therapeutic target for heart failure. Expert Opin Ther Targets. 2018;22:75-83. 